\title{
Modulatory Effects of the Composition and Structure on the Osteogenic Enhancement for Superparamagnetic Scaffolds
}

\author{
Suisui Hao, ${ }^{1 \#}$ Yaoyi Shen, ${ }^{1 \#}$ Haoan Wu, ${ }^{2 *}$ Jie Meng, ${ }^{1}$ Lifei Xie, ${ }^{1}$ Tao Wen, ${ }^{1}$ Ning Gu, ${ }^{2}$ Jian Liu, ${ }^{1 *}$ Yu Zhang ${ }^{2 *}$ and Haiyan $\mathrm{Xu}^{1^{* *}}$
}

Increasing evidence shows that superparamagnetic scaffolds can enhance the osteogenesis under magnetic fields. The aim of this work is to compare the magnetization and the osteogenic enhancement of superparamagnetic scaffolds composed of different compositions with different microstructures. Herein 9 kinds of superparamagnetic scaffolds of PLA, polyurethane and gelatin were fabricated by incorporating iron oxide nanoparticles in polymeric matrices, and using the process of electrospinning, salt-leaching, and solution casting to obtain microstructure of nanofibrous, porous and smooth respectively, while hydroxyapatite nanoparticles were incorporated in all the scaffolds with the same percentage. It was showed that the magnetization behavior of the scaffolds was associated with the composition and microstructure as well as with the osteogenic enhancement of the scaffolds. The nanofibrous scaffold composed of PLA, nHA and MNPs possessed the strongest magnetization, and significantly promoted the osteogenic differentiation of pre-osteoblasts and bone marrow derived mesenchymal stem cells (bMSCs) under magnetic fields, evidenced by the upregulated gene expression of Runx2 and BMP2, the increased ALP activity, OPN and OCN of the cells. The optimal scaffold recruited more bMSCs and enhanced the osteogenic differentiation and the cross talk among the bMSCs, macrophages and fibroblasts under the magnetic field.

Keywords: Superparamagnetic scaffold; Magnetization; Osteogenic differentiation

Received 3 September 2018, Accepted 29 October 2018

DOI: $10.30919 / \mathrm{es} 8 \mathrm{~d} 782$

\section{Introduction}

The bone grafting by using either autografts or allografts has been the "gold standard" treatment for patients suffering from critical bone defects, "though there are drawbacks such as complications or uncomplete integration of bone tissue to the implantations. ${ }^{1,2}$ In order to gain alternative bone graft substitutes, great efforts from tissue engineering and regenerative medicine have been made in the past decades and promising progresses are being achieved steadily. ${ }^{3,4}$ In recent years, we and many other research groups have reported that the incorporation of iron oxide nanoparticles in polymeric or inorganic scaffolds can endow superparamagnetic property to the scaffolds, which significantly enhanced the osteogenesis under the applied magnetic fields both in vitro ${ }^{5,6,7}$ and in vivo, ${ }^{8,9,10,11}$ because the superparamagnetic scaffolds can be effectively magnetized by responding to the magnetic fields and therefore generate microdeformation to apply mechanical forces directly to bone-related cells grown on the scaffolds, ${ }^{12,13}$ including not only mesenchymal stem cells $^{14}$ and pre-osteoblasts, but also macrophages ${ }^{15}$ and fibroblasts ${ }^{9}$

${ }^{1}$ Institute of Basic Medical Sciences Chinese Academy of Medical Sciences, School of Basic Medicine Peking Union Medical College, Beijing 100005, China

2School of Biological Sciences and Medical Engineering, Southeast University, Nanjing 210096, China

"These authors contributed equally to this work

*E-mail:xuhy@pumc.edu.cn; zhangyu@seu.edu.cn;

liujian@ibms.pumc.edu.cn that are closely related to wound healing. These results suggest that the magnetization of scaffolds play important roles in the generation of mechanical forces enhancing osteogenesis.

It should be noticed that the composition and microstructure for the reported superparamagnetic scaffolds for guiding bone regeneration are largely different. At least, nanofibrous scaffolds, ${ }^{5,7,8,14,15,16}$ porous scaffolds, ${ }^{6,1217}$ hydrogels, ${ }^{18,19}$ and even casting films ${ }^{20}$ are involved, at the same time a various kind of materials are employed, such as PLA, ${ }^{5,7,8,15}$ PCL,${ }^{6,17,21}$ chitosan, ${ }^{22,23,24,25}$ collagen, ${ }^{10}$ gelatin, ${ }^{26}$ hydroxyapatite, ${ }^{27,28,29}$ bio-glass. ${ }^{17}$ For some examples, magnetic nanofibrous PLA scaffolds with a low or moderate-intensity magnetic field could enhance the proliferation and osteogenic differentiation of MC3T3-E1 cells, ${ }^{5,7}$ and magnetic nanofibrous composite of chitosan and poly(vinyl alcohol) enhanced the proliferation of MG63 human osteoblast-like cells. ${ }^{23}$ Under the external static magnetic field (SMF), porous scaffold of magnetic $\mathrm{PCL}^{6}$ or $\mathrm{HA}^{29}$ promoted the osteoblast differentiation and bone formation in vitro and significantly enhanced the new bone formation. A magnetic porous scaffold consisting of mesoporous calcium silicate and chitosan promoted osteogenic differentiation of human bone marrow stromal cells. ${ }^{24}$ As for hydrogel scaffolds, a gelatin sponge loading small paramagnetic iron oxide nanoparticles was demonstrated more newly formed bone and a better preserved alveolar ridge in the incisor sockets of Sprague-Dawley rats. ${ }^{26} \mathrm{~A}$ multifunctional magnetic-responsive hydrogel made of methacrylated chondroitin sulfate showed the potential application in tendon-tobone interface. ${ }^{19}$ Besides, a magnetic casting film consisting of Terfenol$\mathrm{D}$ and poly(vinylidene fluoride-co-trifluoroethylene) enhanced the 
proliferation of MC3T3-E1 with magnetoelectric stimuli. ${ }^{20}$ Nevertheless, whether the magnetization property is different among various magnetic scaffolds and associated with the function of enhancing osteogenesis under the magnetic fields are still open questions, which are of implications to design magnetic scaffolds for uses in the guidance of targeting tissues regeneration.

We therefore hypothesized that the magnetization property of scaffolds is dependent on their compositions and microstructures, because the chemical molecules and microstructures of the scaffolds provide different microenvironments to the embedded iron oxide nanoparticles, which would be associated with their function of osteogenic induction. To verify the hypothesis, 9 kinds of superparamagnetic scaffolds were fabricated, and their magnetization property and the function of osteogenesis induction were compared in pre-osteoblasts, from which an optimal scaffold was selected and the function was further verified in bone marrow derived mesenchymal cells of mice.

\section{Materials and Methods}

\subsection{Materials}

The product of $\mathrm{Fe}_{3} \mathrm{O}_{4}$ nanoparticles (MNPs, coated with dimercaptosuccinic acid (DMSA)) was purchased from Nanoeast (Nanjing, China), with an average diameter of $10 \mathrm{~nm}$. The saturated magnetization of MNPs is $69.72 \mathrm{emu} / \mathrm{g} .{ }^{30}$ The product of hydroxyapatite nanoparticles (nHA) was purchased from Nanjing Emperor Nano Material Company (Nanjing, China). Poly (lactic acid) (PLA, PDL-10, $80 \mathrm{KD}$ ) was purchased from Changchun SinoBiomaterials Company (Changchun, China); Gelatin from bovine skin (Gel, G9382, type B, $225 \mathrm{~g}$ bloom) was purchased from Sigma-Aldrich (USA); Polyether polyurethane (PU, WHT-8170, $1.1 \mathrm{~g} / \mathrm{cm}^{3}$ ) was purchased from Yantai Wanhua Polyurethane Co., Ltd (Yantai, China).

\subsection{Preparation of scaffolds}

Scaffolds were prepared using electrospinning technique, salt leaching or solution casting. Three kinds of polymeric materials were selected as the main component in the scaffolds, including PLA, PU and Gel. In each kind of polymeric scaffold, MNPs and nHA were supplemented, in order to allow the scaffolds to be superparamagnetic and bone conductive respectively. The composition, physical characteristics and abbreviation for each kind scaffold were summarized in Table 1 . The detail of fabrication procedure was described below and an example was also provided in the supplementary information.

In order to conduct cellular experiments with the scaffolds, the material samples were trimmed to round sheets and subjected to ethylene oxide sterilization, the diameter was the same as the wells of culture plates, allowing the wells' bottom completely covered with the sample. Scaffolds with $5 \%, 10 \%$ and $15 \%$ MNPs (w \% of polymer) were used to determine the saturated magnetization of MNPs, and scaffolds with $10 \%$ MNPs (w \% of polymer) were used for all cell biology experiments.

\subsection{Preparation of nanofibrous scaffolds:}

A certain amount of MNPs and nHA was added in the solvent of Dimethylacetamide (DMAc), 2, 2, 2-trifluoroethanol (TFEA) or Hexafluoroisopropanol (HFIP) and sonicated by using an ultrasonic probe for $10 \mathrm{~min}$ (work and stop for $2 \mathrm{~s}$ alternately). PU, PLA or Gel was dissolved in the DMAc, TFEA or HFIP solution respectively. The resulting solutions were subjected to the electrospinning device with optimized parameters. The scaffolds of Gel were crosslinked by $0.5 \%$ of glutaraldehyde. The residue of glutaraldehyde was removed completely by immersing the samples in the glycine solution for $6 \mathrm{~h}$ and repeating four times. All the samples were dried in the vacuum oven for $48 \mathrm{~h}$ at the room temperature.

\subsection{Preparation of porous scaffolds:}

A certain amount of MNPs and nHA were added to Tetrahydrofuran (THF) or Dimethyl sulfoxide (DMSO) and sonicated by using an ultrasonic probe for $10 \mathrm{~min}$ (work and stop for $2 \mathrm{~s}$ alternately). PU or PLA was dissolved in the THF solution, and Gel was dissolved in the DMSO solution. The solutions were poured into glass dishes where $\mathrm{NaCl}$ particles of $75-150 \mu \mathrm{m}$ in diameter were spread on the bottom. For the solution containing Gel, $0.5 \%$ glutaraldehyde was added in the solution prior to the casting. After 2 days, the samples were washed by distilled water for 4 times to remove the $\mathrm{NaCl}$ particles.

Table 1. The composition, physical characteristic and abbreviation for scaffolds.

\begin{tabular}{|c|c|c|c|c|c|c|}
\hline Scaffold & Morphology & Fabrication $^{*}$ & Solvent & $\begin{array}{c}\text { Polymer } \\
\text { concentration (wt } \% \\
\text { in solutions) }\end{array}$ & $\begin{array}{c}\text { nHA } \\
\text { (wt\%of } \\
\text { polymer) }\end{array}$ & $\begin{array}{c}\text { MNPs } \\
\text { (wt\%of } \\
\text { polymer) }\end{array}$ \\
\hline mag-PLA/NF & Nanofibrous & ES & TFEA & PLA, $30 \%$ & \multirow{3}{*}{50} & \multirow{3}{*}{$\begin{array}{c}5 \text { or } 10 \text { or } \\
15\end{array}$} \\
\hline mag-PLA/Poro & Porous & SL & THF & PLA, $10 \%$ & & \\
\hline mag-PLA/Sm & Smooth film & $\mathrm{SC}$ & THF & PLA, $10 \%$ & & \\
\hline mag-PU/NF & Nanofibrous & ES & DMAc & PU, $10 \%$ & \multirow{3}{*}{50} & \multirow{3}{*}{$\begin{array}{c}5 \text { or } 10 \text { or } \\
15\end{array}$} \\
\hline mag-PU/Poro & Porous & SL & THF & PU, 10\% & & \\
\hline mag-PU/Sm & Smooth film & $\mathrm{SC}$ & THF & PU, $10 \%$ & & \\
\hline $\mathrm{mag}-\mathrm{Gel} / \mathrm{NF}$ & Nanofibrous & ES & HFIP & Gel, 10\% & \multirow{3}{*}{50} & \multirow{3}{*}{$\begin{array}{c}5 \text { or } 10 \text { or } \\
15\end{array}$} \\
\hline mag-Gel/Poro & Porous & SL & DMSO & Gel, $5 \%$ & & \\
\hline mag-Gel/Sm & Smooth film & $\mathrm{SC}$ & $\mathrm{dd}-\mathrm{H}_{2} \mathrm{O}$ & Gel, $5 \%$ & & \\
\hline
\end{tabular}

*ES: electrospinning; SL: salt leaching; SC: solution casting 
For the samples containing Gel, the excess glutaraldehyde was removed by immersing in the glycine solution for 4 times additionally. The scaffolds of Gel were freeze-dried for $48 \mathrm{~h}$; the scaffolds of PU or PLA were purified in the vacuum oven for $48 \mathrm{~h}$ at the room temperature.

\subsection{Preparation of smooth scaffolds:}

A certain amount of MNPs and nHA was added to THF or double distilled water $\left(\mathrm{dd}-\mathrm{H}_{2} \mathrm{O}\right)$ and sonicated by using an ultrasonic probe for $10 \mathrm{~min}$ (work and stop for $2 \mathrm{~s}$ alternately). PU or PLA was dissolved in the THF solution, and Gel was dissolved in the dd- $\mathrm{H}_{2} \mathrm{O}$ solution. The solutions of PU or PLA were poured into glass dishes, letting the solvent to vaporize at the room temperature. For the solution containing Gel, $0.5 \%$ glutaraldehyde was added in the solution prior to the casting and letting the solvent to vaporize at 50 ${ }^{\circ} \mathrm{C}$ in oven. The excess glutaraldehyde was removed by immersing in the glycine solution for 4 times. All the samples were dried in the vacuum oven for $48 \mathrm{~h}$ at the room temperature.

\subsection{Electron microscope observation and magnetism analysis of scaffolds}

Scanning electron microscope (SEM, SU-8010) was applied to observe the morphology of the scaffolds. For the nanofibrous scaffolds, the average fiber diameter was counted from 100 random selected fibers in SEM pictures and measured by ImageJ software. The MNPs in the fibers were observed with transmission electron microscopy (TEM, JEM-1400 Plus).

The M-H curves of the scaffolds were obtained by a vibrating sample magnetometer (VSM, Lakeshore VSM 7407). The saturated magnetization of MNPs per gram in the scaffold $\left(\mathrm{SM}_{\mathrm{MNP}}\right)$ was calculated as:

$\mathrm{SM}_{\mathrm{MNP}}=\mathrm{SM} / \mathrm{W}_{\mathrm{MNP}}$

where $\mathrm{SM}$ is the saturated magnetization of scaffold, $\mathrm{W}_{\text {MNPs }}$ is the weight of the MNPs in the scaffold.

The average of $\mathrm{SM}_{\mathrm{MNP}}\left(\mathrm{SM}_{\mathrm{MNP}}\right)$ for the scaffolds was calculated as:

$$
{ }_{\mathrm{a}} \mathrm{SM}_{\mathrm{MNP}}=\left(\mathrm{SM}_{\mathrm{MNP} \%}+\mathrm{SM}_{\mathrm{MNP10} \%}+\mathrm{SM}_{\mathrm{MNP15 \%}}\right) / 3
$$

\subsection{Mechanical test}

The film samples prepared from different kinds of polymer with the weight ratio of polymer to $\mathrm{nHA}$ to MNPs $=10: 5: 1$ were cut into rectangular pieces $(50 \mathrm{~mm} \times 10 \mathrm{~mm})$. The thickness of Gel, PLA, and PU was $1.5 \mathrm{~mm}, 0.20 \mathrm{~mm}$, and $0.25 \mathrm{~mm}$ respectively. A tensile test for each sample was carried out using a universal mechanical testing machine (AG-1S, Shimadzu, Japan) at a crosshead speed of $20 \mathrm{~mm} / \mathrm{min}$. The load cell for Gel, PLA, and PU was $10 \mathrm{~N}, 50 \mathrm{~N}$, and $50 \mathrm{~N}$ respectively. The Young's modulus (E) was calculated from the average slope of stress-strain curve during the range of elongation from $2 \%$ to $6 \%$.

\subsection{Setup of static magnetic field}

A permanent magnet was set under the cell culture plate. The intensity of magnetic fields was obtained and measured according to our previous method described. ${ }^{9}$ The magnetic field was applied to the cells in an interval of $12 \mathrm{~h}$ during the incubation.

\subsection{Cell culture}

The mouse cell lines including pre-osteoblast cell (MC3T3-E1), fibroblast (NIH3T3) and macrophage cell (RAW264.7) were purchased from the Cell Center of Institute of Basic Medical
Sciences, Chinese Academy of Medical Sciences. Pre-osteoblasts were cultured in basal $\alpha$-MEM and $10 \%$ FBS and $0.1 \%$ penicillinstreptomycin. Fibroblast and macrophage was maintained in DMEM containing 10\% FBS, $4500 \mathrm{mg} / \mathrm{L}$ glucose, $4 \mathrm{mM} \mathrm{L}$-glutamine, and $0.1 \%$ penicillin-streptomycin.

Mouse bone mesenchymal stem cells (bMSCs) were isolated from tibia of C57BI/6 mice of about 3-4 weeks old, and characterized by $\mathrm{Sca} 1^{+} \mathrm{CD} 45^{-}$markers via flow cytometry testing. bMSCs were cultivated in the mesenchymal stem cell medium (ScienCell Research Laboratories, San Diego, CA).

\subsection{Cell viability Assay (CCK-8)}

Pre-osteoblasts of $8 \times 10^{3} /$ well or bMSCs of $1 \times 10^{4} /$ well were seeded in a 96-well culture plate, the scaffold samples were fixed in the bottom of wells. After incubation, the culture medium was replaced with the reagent of Cell Counting Kit 8 (CCK-8, Dojindo) in fresh culture medium according to the manufacturer's instruction. The supernatant of each well was collected and measured at $450 \mathrm{~nm}$ by a microplate reader (Epoch R, BioTek).

\subsection{ALP Assay}

Pre-osteoblasts $\left(2 \times 10^{5}\right)$ or Mouse bone mesenchymal stem cells (bMSCs) $\left(3 \times 10^{5}\right)$ were seeded in scaffolds in a 12 -well culture plate and cultivated in the inductive medium with $10 \mathrm{nM}$ dexamethasone, $10 \mathrm{mM} \beta$-glycerophosphate, and $50 \mathrm{mg} / \mathrm{mL}$ ascorbic acid in $\alpha$-MEM medium. The culture plate was set in the magnetic field for designated time. The pre-osteoblasts were lysed in the RIPA cell lysis buffer (Beyotime), the concentration of the lysates was determined by BCA protein assay (Pierce Biotechnology). The lysate samples were diluted to $0.5 \mathrm{mg} / \mathrm{mL}$. After incubation the supernatants of bMSCs cultivation were collected and concentrated to $1 / 5$. The mouse alkaline phosphatase (ALP) ELISA kit (enzyme-linked immunosorbent assay Beyotime) was used to quantify the activity of ALP for the lysates and supernatants according to the manufacturer's instruction.

\subsection{Western Blot}

The pre-osteoblasts $\left(2 \times 10^{5} /\right.$ well $)$ or bMSCs $\left(3 \times 10^{5} /\right.$ well $)$ were cultured in a 12-well culture plate with composites fixed in the wells, and cultivated for the designated time with applied magnetic field in the inductive medium. Then cells were washed and lysed. The osteocalcin (OCN), osteopontin (OPN) in the cell lysate were analyzed by western blot assay using corresponding antibody to OCN, OPN (Abcam) and $\beta$-actin (Sigma-Aldrich) as described previously. ${ }^{9}$ The density of the western blot bands was quantified by using imageJ software (https://imagej.nih.gov/ij/index.html, NIH, Bethesda, MD) and normalized to $\beta$-actin.

\subsection{RT-PCR Analysis}

Cells of $2 \times 10^{5}$ /well were seeded on 12-well plates that fixed with composites to allow the adherence overnight, and cultivated for the designated time with applied magnetic field in the inductive medium. After incubation the cells were collected and RNA was extracted by using Trizol. The concentration of RNA was determined by using a Nanodrop ND1000. The ratio of the absorbance at $260 \mathrm{~nm}$ to that at $280 \mathrm{~nm}$ was detected within 1.9 to 2.1 . The cDNA synthesis was prepared according to the manufacturer's instruction (Invitrogen). The gene expression was analyzed by Quantitative RT-PCR (Biorad connect). The expression of BMP2 and Runx2 was normalized to GAPDH. The mean $\mathrm{C}_{\mathrm{T}}$ values of the samples were calculated. The 
Table 2. The primers serials used in experiments.

\begin{tabular}{lcc}
\hline Target gene & Forward & Reverse \\
\hline Runx2 & 5'-AGGGACTATGGCGTCAAACAG-3 & 5'-CTCGGCGGAGTAGTTCTCATC-3, \\
BMP2 & 5'-GACGTCCTCAGCGAATTTG -3' & 5'-GAGAAACTCGTCACTGGGG-3', \\
GAPDH & 5'-GTCAAGCTCATTTCCTGGT -3' & 5'-CCAGGGTTTCTTACTCCTTG -3' \\
\hline
\end{tabular}

three primers were synthesized by Sangon Biotech (Table 2).

\subsection{Preparation of conditioned media}

Macrophages $\left(2 \times 10^{5} /\right.$ well $)$ or fibroblasts $\left(2 \times 10^{5} /\right.$ well $)$ were seeded in mag-PLA/NF fixed in a 12-well culture plate. After incubated with or without magnetic field of $10 \mathrm{mT}$, the supernatant in each well was collected. The supernatants from the macrophages and from the fibroblasts were mixed at 1:1 (volume ratio). The mixture was then added to equal volume of inductive medium, preparing the conditioned medium (CMs), named as CM@mag-PLA/NF, or CM@mag-PLA/NF+MF.

\subsection{Induction of osteogenesis using conditioned medium}

The bMSCs were seeded at $8 \times 10^{4} /$ well in a 24 -well plate. When the cell monolayer reached $70-80 \%$ of confluence, the culture medium was replaced with fresh normal medium, inductive medium, CM@mag-PLA/NF, or CM@mag-PLA/NF+MF, and was replaced every 3 days. After cultivation for 21 days, Alizarin Red S (ARS) staining assay was applied to detect mineralized calcium deposition of bMSC cells. The stained cells were then incubated with $10 \%$ (w/v) sodium dodecyl sulfate (SDS) at $37{ }^{\circ} \mathrm{C}$ overnight. The absorbance at $405 \mathrm{~nm}$ of the cell lysates was measured by using microplate reader.

\subsection{Transwell Assay}

The bMSCs $\left(2 \times 10^{5}\right.$ cells $)$ were added in the upper well of the transwell (Corning, $0.5 \mu \mathrm{m}$ ) for $24 \mathrm{~h}$ incubation. Different $\mathrm{CM}$ of $800 \mu \mathrm{L}$ was added in the lower chamber. The upper surface of the well was carefully swabbed to eliminate the cells when the incubation was finished, and the upper chamber was washed and fixed with $4 \%$ paraformaldehyde. After rinsing twice, the cells were stained by $0.1 \%$ crystal violet for $1 \mathrm{~h}$, followed by being dried in the air. The migrated cells were observed with microscope (Olympus IX71) as well as measure the absorbance of crystal violet at $570 \mathrm{~nm}$ with a microplate reader (Biotek).

\subsection{Statistical Analysis}

The data shown were obtained from at least 2 independent experiments; triplicate was set for each experiment. Data were expressed as mean \pm standard error of the mean (SEM). The twotailed student's t-test was applied for the statistical analysis (Microsoft software, 2016). A value of $\mathrm{p}<0.05$ was considered significance between conditions.

\section{Results}

\subsection{Physicochemical characterization and magnetization behavior}

\section{of scaffolds}

In the current study, 9 kinds of scaffold were prepared using different chemical compositions and processing techniques, which was illustrated by a schematic graph (Fig. 1A). Morphological structures of the resulting scaffolds were observed using scanning electron microscopy (SEM) and shown in Fig. 1B. As shown that in the nanofibrous scaffolds there were numerous nanofibers crossing with each other, the diameter of the fibers was similar and around $200.2 \pm 56.7 \mathrm{~nm}$ in mag-PLA/NF, $179.7 \pm 57.0 \mathrm{~nm}$ in mag-PU/NF and $187.6 \pm 66.3 \mathrm{~nm}$ in mag-Gel/NF; the three kinds of porous scaffolds showed good porosity, and no significant structural differences were observed among the porous scaffolds that made of different materials; and the casting films exhibited smooth plane-like structure. Scaffolds prepared from different polymeric components (PLA, PU, and gelatin) showed different mechanical properties. PLA is one plastic material with relative higher mechanical strength than $\mathrm{PU}$, while PU is highly elastic, and Gel is one soft hydrogel (Figure 1C).

All of the scaffolds possessed superparamagnetic performance, they could be magnetized quickly when the magnetic field (MF) was applied, and displayed an almost immeasurable coercive force and remnance when the MF was removed. The magnetic hysteresis loops for the three nanofibrous magnetic scaffolds were presented in Fig. $2 \mathrm{~A}$. The saturated magnetization of iron oxide nanoparticles (MNPs) in the 9 scaffolds was calculated and summarized in Fig. 2B, which could reflect the magnetization behavior of the scaffolds. From the comparison of physical microstructures, MNPs in the nanofibrous PLA exhibited much stronger saturated magnetization than in the smooth and porous one; while that in the nanofibrous PU or nanofibrous Gel showed similar saturated magnetization with smooth PU or smooth Gel, and the saturated magnetization of MNPs in the porous PU or porous Gel was the lowest among the three PU scaffolds or among the three Gel scaffolds respectively. From the comparison of chemical composition, the saturated magnetization of MNPs in the nanofibrous PLA was higher than that of MNPs in the nanofibrous PU and nanofibrous Gel, while that of MNPs in the smooth PU was higher than that of MNPs in the smooth PLA and Gel, and that of MNPs in the porous PU was higher than that of MNPs in the porous PLA and porous Gel. Taken above together, it was suggested that (1) the saturated magnetization of mag-PLA was much more dependent on the physical structure of scaffolds than mag-PU and mag-Gel; (2) porous structure weakened the magnetization for the scaffolds compared with the nanofibrous and smooth structure; and (3) the saturated magnetization of mag-Gel was lower than that of mag-PU and mag-PLA, no matter what microstructure it was (Fig. 2B).

To evaluate the cell compatibility of the scaffolds, pre- 
A

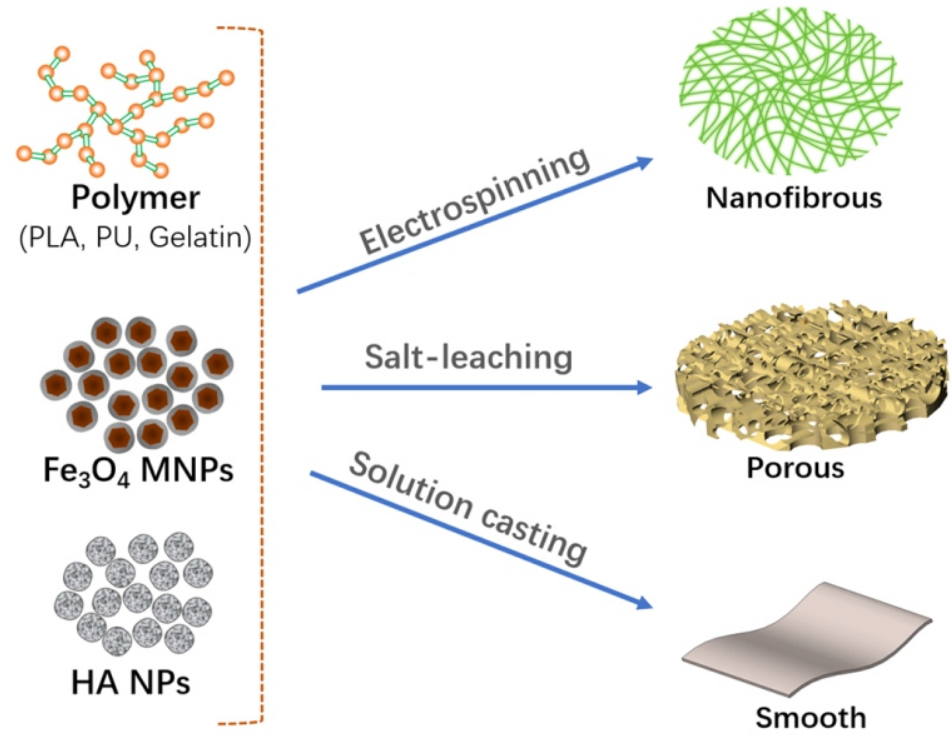

B

Nanofibrous

Porous

Smooth

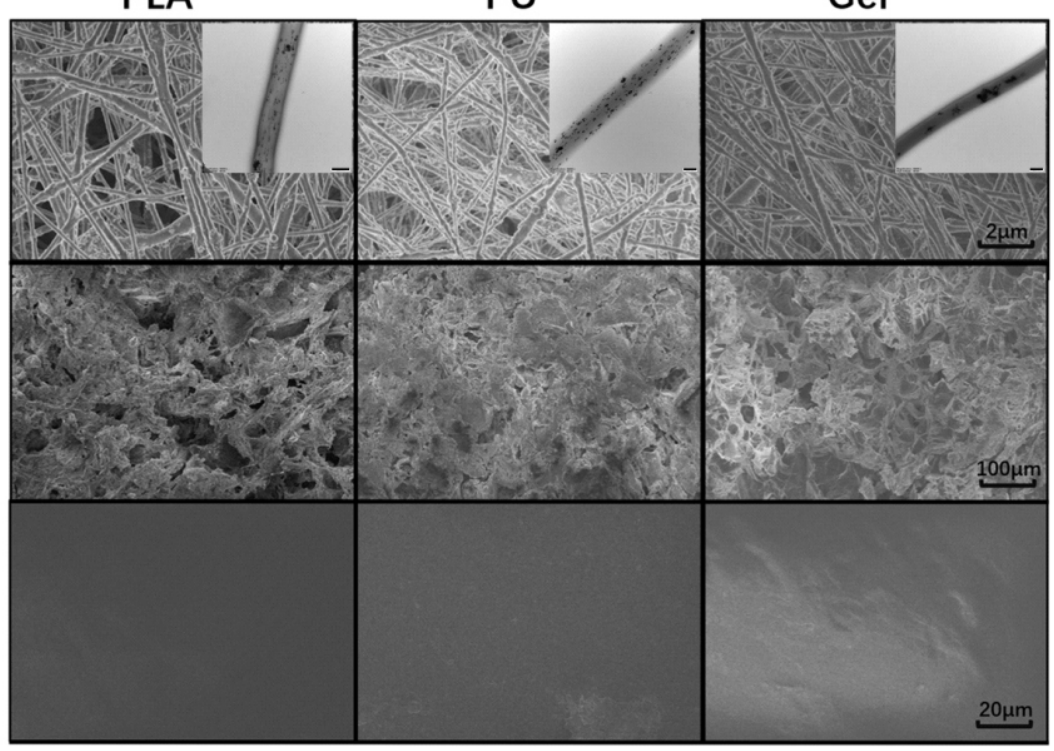

C

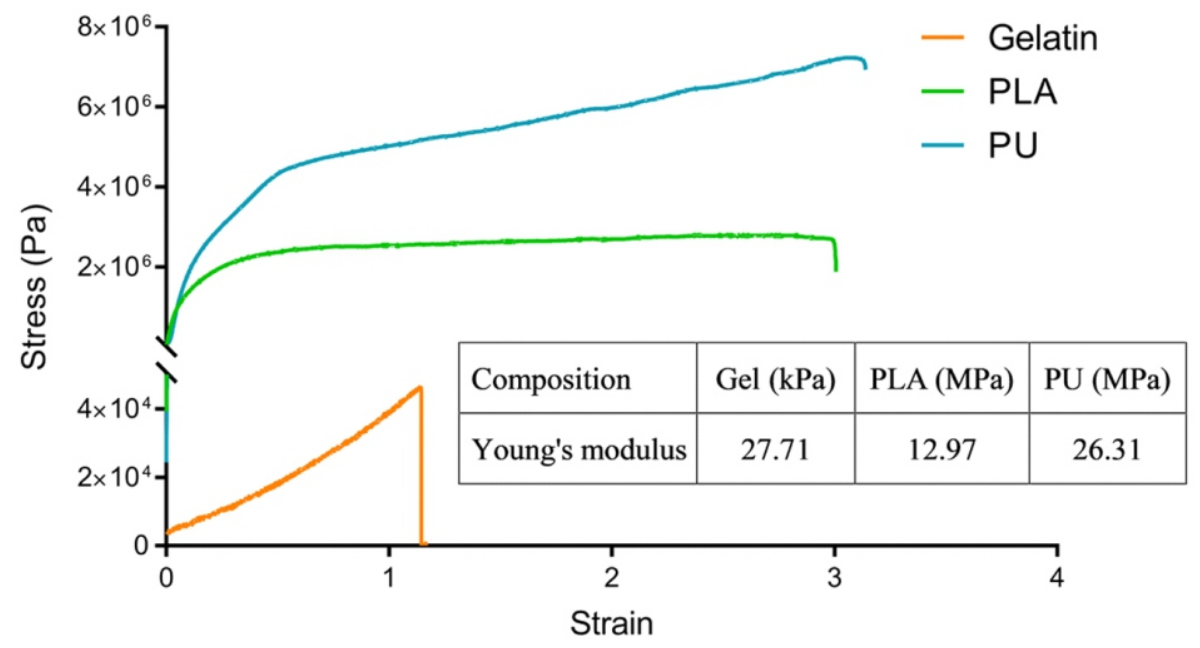

Fig. 1 Morphological characterization of scaffolds. (A) Schematic graph of the preparing process for the different scaffolds. (B) Representative scanning electron microscopy image and transmission electron microscopy (embedded pictures, scale bar: $200 \mathrm{~nm}$ ) of the scaffolds. (C) Stress-strain curve and the stiffness (Young's modulus) of film samples prepared from different kinds of polymer. The weight ratio of polymer to nHA to MNPs was 10:5:1 for all scaffolds. 
A
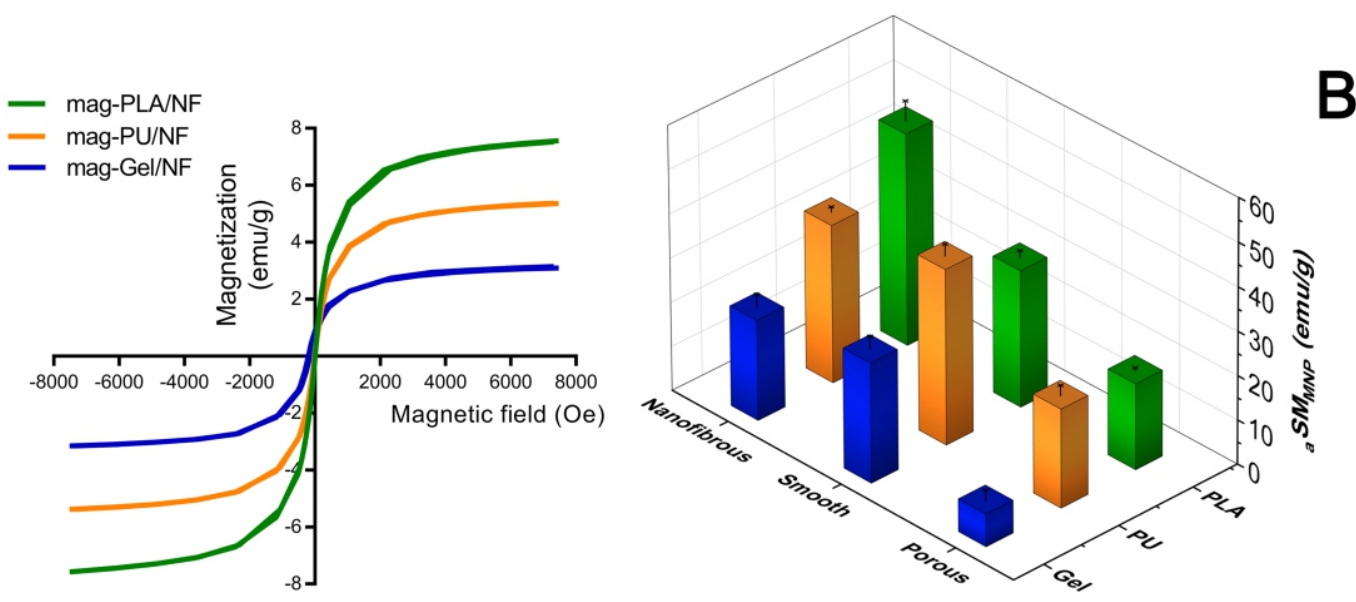

C
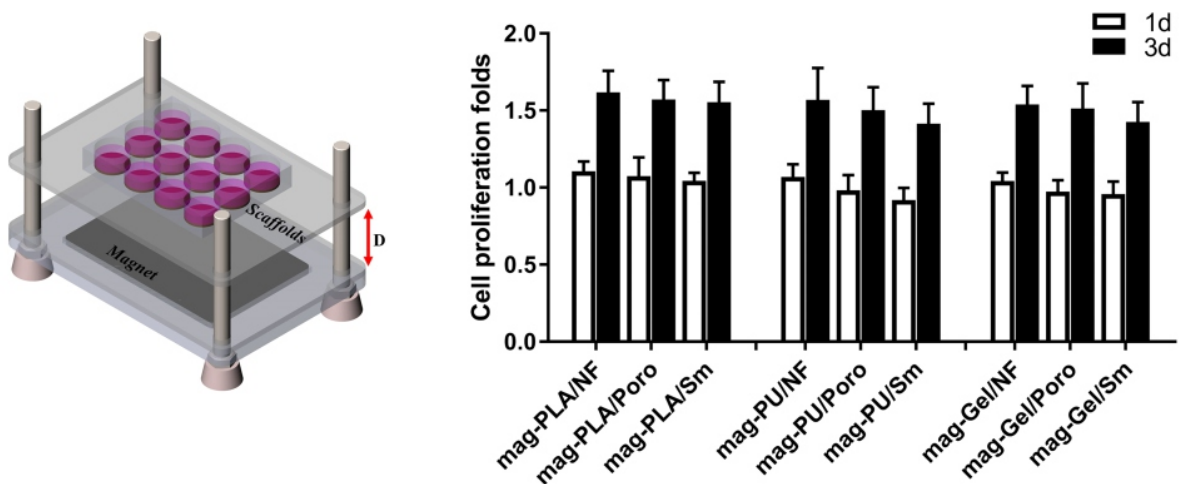

Fig. 2 The superparamagnetic responsive profile of different scaffolds. (A) Representative magnetization curves of three kinds of nanofibrous scaffolds with $15 \%$ MNPs. (B) The summary of the average saturated magnetization of MNPs in the scaffolds $\left({ }_{\mathrm{a}} \mathrm{SM}_{\mathrm{MNP}}\right)$. The statistical results between each two groups were listed in Table S2. (C) The schematic graph of culture system applied with the magnetic field. (D) The viability of pre-osteoblasts grown on different scaffolds under the magnetic fields of $10 \mathrm{mT}$ within 3 days. Cells cultured on PU film in the absence of MF (data not shown) were selected as control group.

osteoblasts were grown on the 9 scaffolds under the MF of $10 \mathrm{mT}$ (Fig. 2C), and the pre-osteoblasts could proliferate normally in the incubation of $72 \mathrm{~h}$ (Fig. 2D), indicating that all the scaffolds supported the cells growth under the magnetic field. There was not significant difference in the groups with the same time period of culture, indicating that the biocompatibility for all those scaffolds was similar. In the following experiments for the pre-osteoblasts, 10 $\mathrm{mT}$ was applied to the cellular culture system.

\subsection{Effects of the scaffold magnetization on the function of enhancing osteogenesis of pre-osteoblasts}

Next we investigated whether the scaffolds with different magnetization behaviors were associated with the osteogenic enhancement on pre-osteoblasts. It has been demonstrated that scaffolds with the superparamagnetic property significantly enhanced osteogenesis in vitro and in vivo when compared with scaffolds without the superparamagnetic property. ${ }^{5,6,8,10,31}$ The aim of this study is to figure out the difference of scaffolds with different polymeric component and morphology in the osteogenic enhancement. Therefore, scaffolds without iron oxide nanoparticles were not set in this study. It was seen that under the applied magnetic field of $10 \mathrm{mT}$, mag-PLA exhibited obvious advantage to enhance the expression of Runx 2 than mag-PU and mag-Gel, no matter what microstructures. As for the expression of BMP2, when the polymer content was PLA or Gel, the nanofibrous structure showed stronger enhancement than smooth and porous; and when the polymer content was PU, the effect of the microstructures was not significant, though the nanofibrous one exhibited a slight up-regulation. In particular, mag-PLA/NF induced the highest level of Runx2 and BMP2 among the 9 scaffolds Fig. 3A\&B. Similarly, mag-PLA in each kind of the microstructures induced higher levels of ALP activity than mag-PU and mag-Gel in the corresponding microstructures, and mag-PLA/NF induced the highest one (Figure $3 \mathrm{C})$. It was also noticed that in the absence of the applied magnetic field, the effects of scaffolds on the gene expression of Runx 2 and BMP2 were weaker than those under the magnetic field, while the difference of ALP activity in the presence and absence of magnetic field was only observed in the three scaffolds of nanofibrous structure. Taken above together, mag-PLA/NF was the optimal one among the 9 scaffolds in the osteogenic enhancement; and BMP2 was more sensitive to the scaffold composition and microstructure than Runx2 and ALP. It has been widely recognized that BMP2 is a very important growth factor in the osteogenesis, especially in the initial period, while runx2 is a downstream pathway initiated by BMP2. ${ }^{32}$ Here BMP2 and runx2 in gene level was examined on the day 3 of incubation, therefore BMP2 showed relative higher sensitivity because it was a very early stage of bone differentiation.

Osteopontin (OPN) is one typical extracellular protein secreted by osteoblasts in the late stage of osteogenic differentiation. Preosteoblasts grown on mag-PLA/NF under the magnetic field of 10 


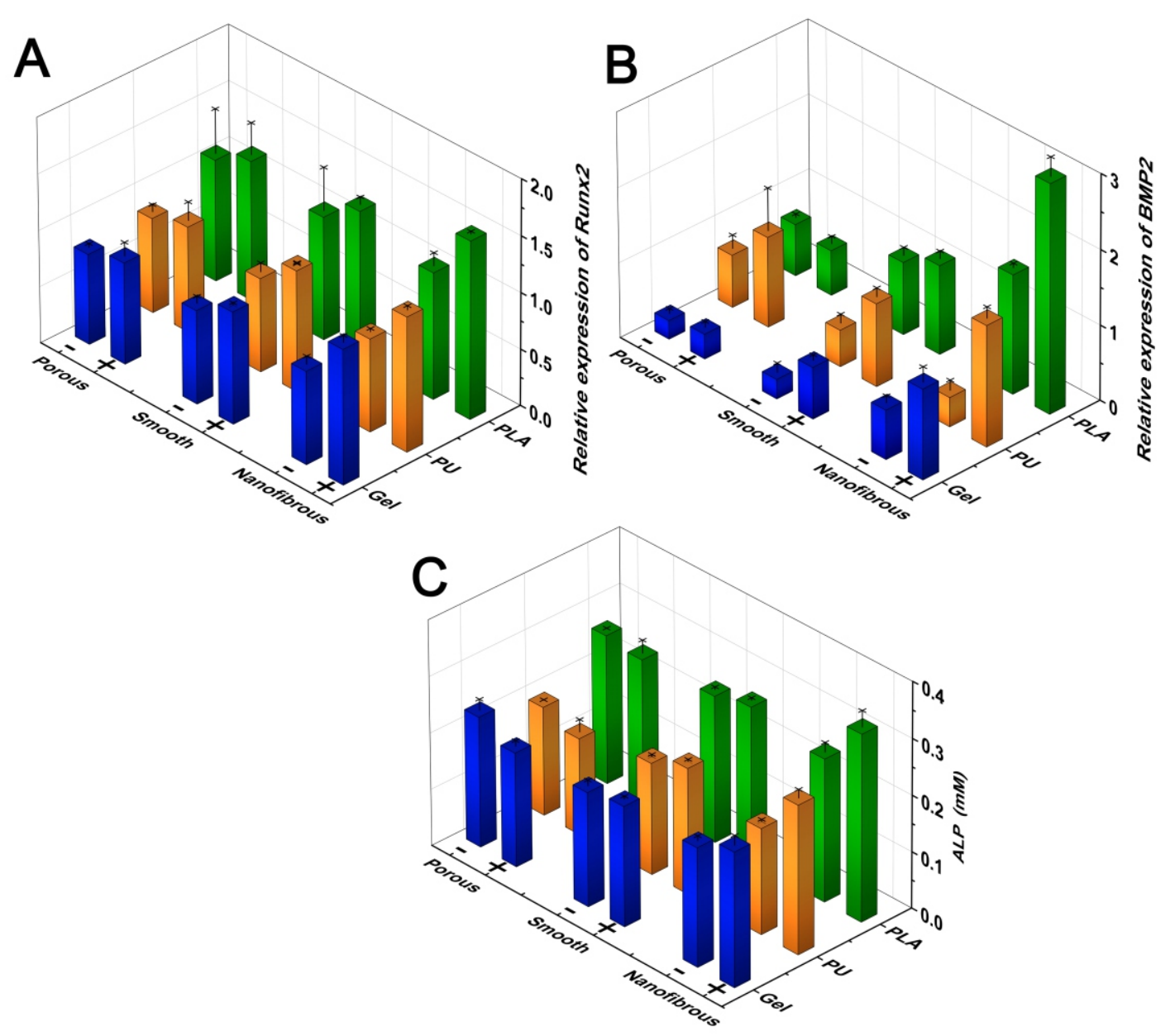

Fig. 3 Effects of composition and morphological structure for the scaffolds on the expression of Runx2 (A) and BMP2 (B) and the activity of ALP in total protein (C) for pre-osteoblasts with or without the magnetic field. "+" refers to under the magnetic field of 10 $\mathrm{mT}$, and "-" refers to in the absence of the magnetic field. The incubation time was $72 \mathrm{~h}$. The statistical results between each two groups were listed in Table S3.

mT (MF10) expressed a higher level of OPN than those on magPLA/Poro and mag-PLA/Sm (Fig. 4A); meanwhile, the expression was also higher than that by the cells on mag-PU/NF and magGel/NF under MF10 (Fig. 4B). These results provided further support that the higher saturated magnetization the stronger enhancement to the osteogenesis, and therefore mag-PLA/NF was chosen as an ideal scaffold to induce osteogenesis and bone regeneration.

\subsection{The mag-PLA/NF under magnetic field promoted the} osteogenic differentiation of bMSCs

To further verify the enhancement effect of mag-PLA/NF on the osteogenesis under the magnetic fields, bone marrow derived mouse mesenchymal stem cells (bMSCs) were seeded in mag-PLA/NF in the inductive medium and different magnetic field. bMSCs can proliferate normally in all groups and there was not significant difference in the groups at the same incubation time (Fig. 5A). After incubation for $7 \mathrm{~d}$, results from RT-PCRs showed that mag-PLA/NF with MF5 or MF10 could significantly enhance the expression of Runx2 in reference to mag-PLA/NF without MFs (Fig. 5B), and the expression of BMP2 was upregulated as well under the same conditions (Fig. 5C). At the same time, mag-PLA/NF with MF10 increased the ALP activity significantly (Fig. 5D).

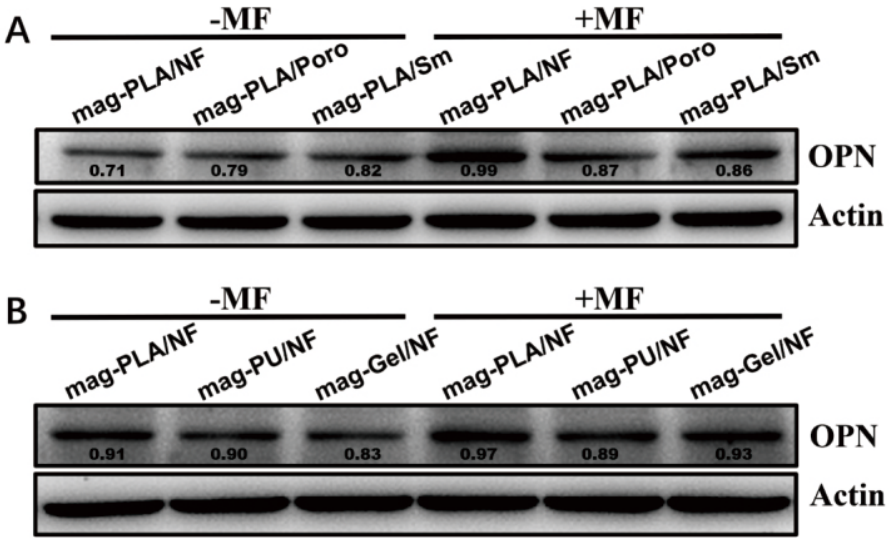

Fig. 4 The expression of OPN for pre-osteoblasts grown on magPLA with different microstructures (A) or on the nanofibrous scaffolds with different chemical compositions (B), in the absence of magnetic field (-MF) or in the presence of magnetic field (+MF) for 21 day incubation. The densities of the OPN bands were quantified using imageJ software and normalized to $\beta$-actin. 

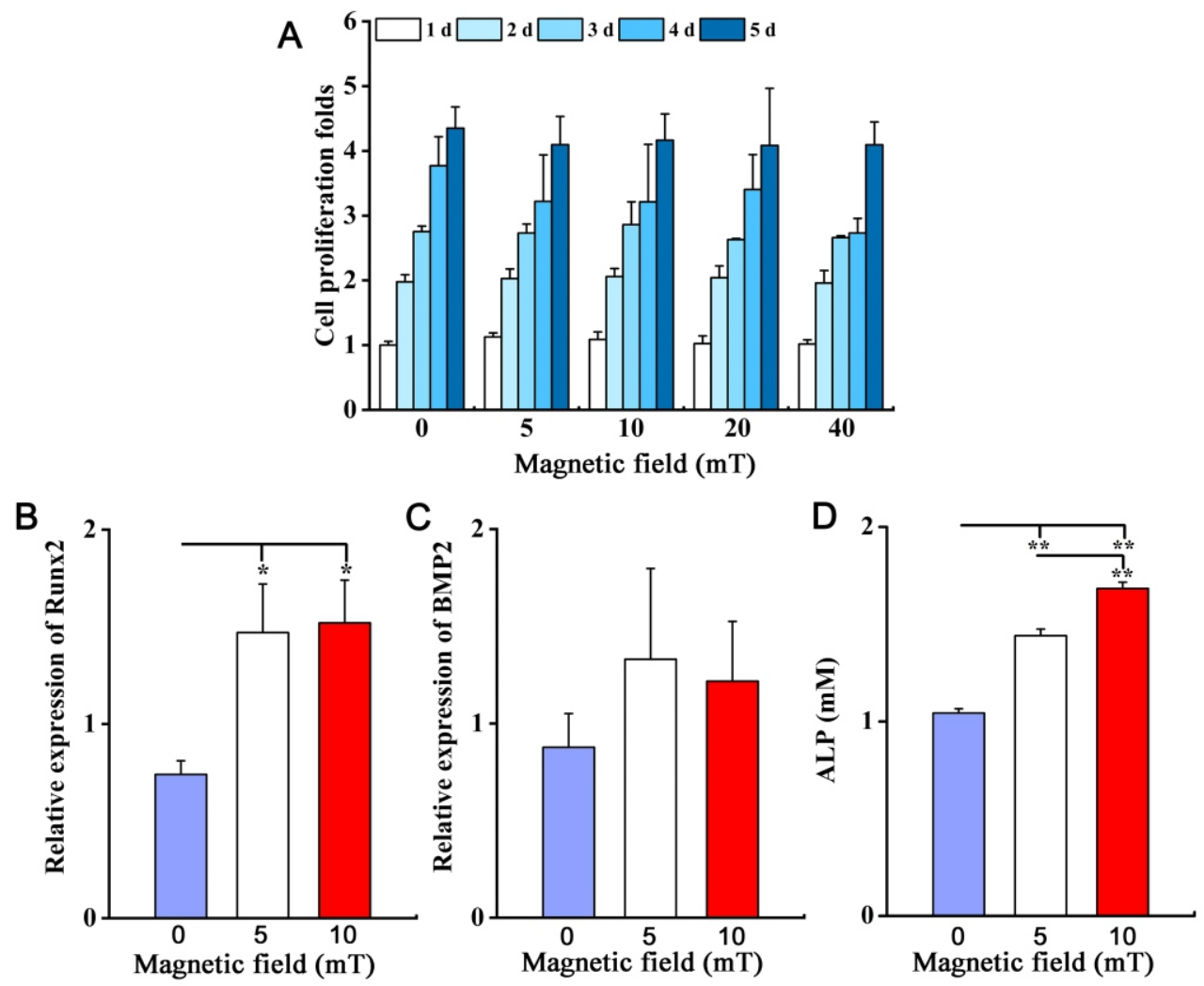

Fig. 5 The synergy effect of mag-PLA/NF with different field strength of MFs on MSCs. (A)The cell proliferation of MSCs. (B) Runx2 and (C) BMP2 genes expression of MSCs grown on mag-PLA/NF with MFs, (D) ALP activity of MSCs grown on magPLA/NF with MFs for 10 days.

Western blot analysis showed that bMSCs cells grown on mag$\mathrm{PLA} / \mathrm{NF}+\mathrm{MFs}$ displayed higher expression of OCN and OPN than those grown on the mag-PLA/NF without MFs. The effect became stronger when the incubation time was prolonged from 14 days to 21 days (Fig. 6).

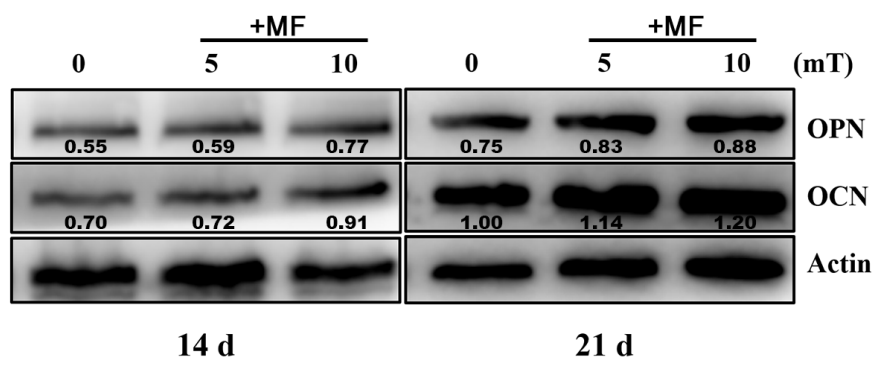

Fig. 6 The osteogenic effect of mag-PLA/NF with MFs on MSCs. OCN and OPN levels of MSCs grown on mag-PLA/NF with MFs for (A) 14 days and (B) 21 days. The densities of the OPN and OCN bands were quantified using image J software and normalized to $\beta$ actin.

3.4 The mag-PLA/NF under the magnetic fields facilitated the cross talk of bMSCs with macrophages and fibroblasts

We previously demonstrated that both macrophages and fibroblasts grown on mag-PLA/NF secreted higher amounts of growth factors including VEGF, PDGF and bFGF, ${ }^{9,15}$ here we prepared conditioned media (CMs) by mixing the inductive medium and culture supernatants from the incubation of macrophages and fibroblasts grown on mag-PLA/NF under MF5 or MF10 or in the absence of MF at 1:1 (volume ratio). The CMs were employed in the bottom chamber of a transwell device. It was seen that more MSCs were recruited from the upper chamber into the bottom chamber after $24 \mathrm{~h}$ of incubation with CM@mag-PLA/NF+MF (5 mT and $10 \mathrm{mT}$ ), compared with that with CM@mag-PLA/NF (Fig. 7), and the highest amount of cells were recruited by CM@mag-PLA/NF+MF10.

The osteogenic differentiation of bMSCs induced by different media was determined by Alizarin Red S staining used to depict bone mineralization by orange-red staining of calcium-based mineral deposits and hydroxyapatite crystals formed during the later phases of osteoblast maturation. It was seen that MSCs incubated in CM@mag-PLA/NF+MF10 differentiated into osteoblasts much faster (bottom right of Figure 8A) than those in CM@mag-PLA/NF without the applied magnetic field (bottom left of Fig. 8A). The enhancement effect induced by CM@mag-PLA/NF+MF10 was even better than that induced by the inductive medium control (upper right of Fig. 8A). The quantification data were shown in Figure 8B.

\section{Discussion}

The guidance of bone tissue regeneration and repair for defects that are larger than the critical size has been one of the major challenges in biomaterials science, because of the increased need of tissue replacement in traumas, tumor excision, skeletal abnormalities or 


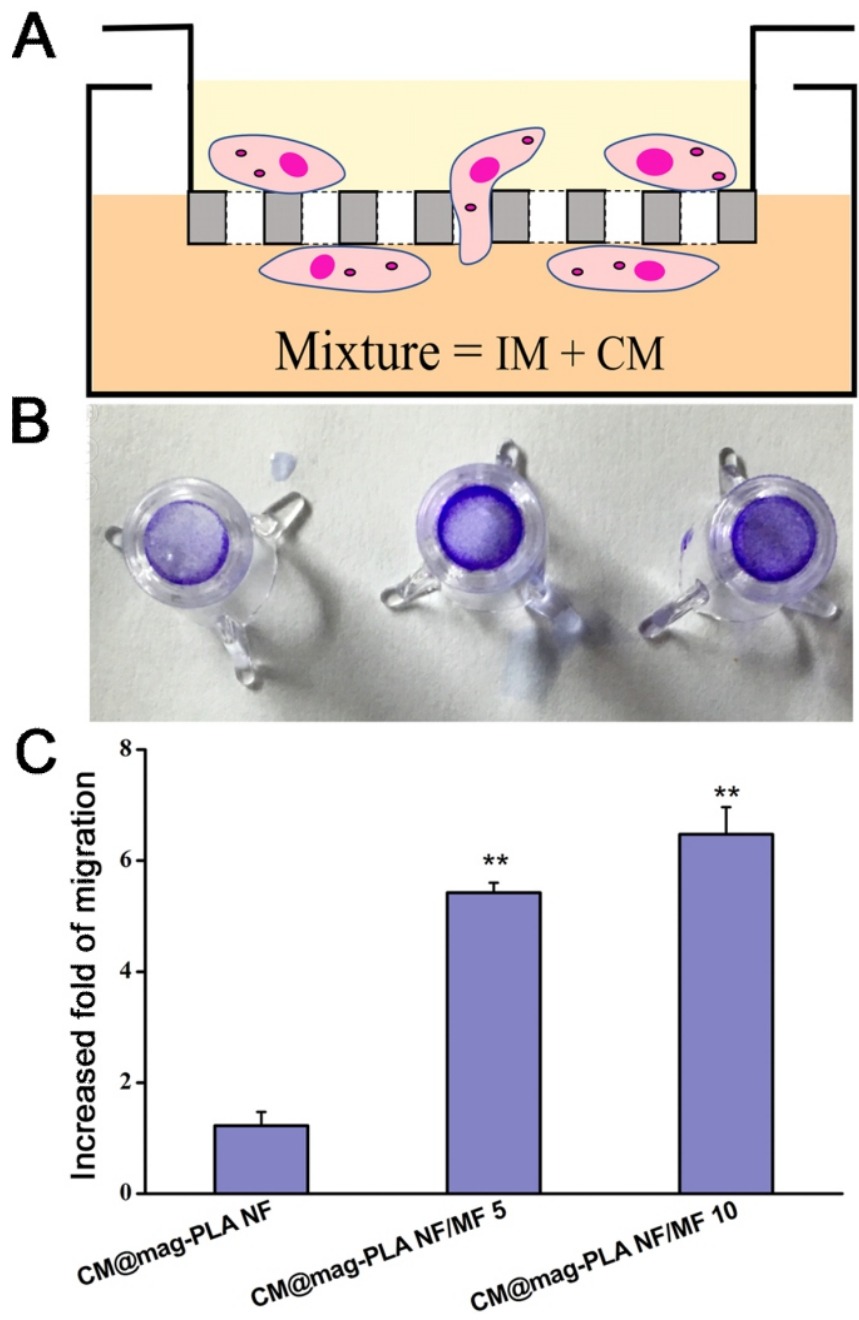

Fig. 7 The migration of bMSCs induced by conditioned media (CMs). (A) The schematic graph of transwell. (B) Photographs from transwell assay showing the intensity of crystal violet. (C) Increased folds of the migration quantified with (B). resection. As osteocytes are mechano-sensitive cells, mechanical force stimuli have been used to improve bone regeneration previously. ${ }^{33-34}$ Recent years, an approach of superparamagnetic scaffolds in combination with static magnetic fields has attracted increasing interests for research into bone tissue engineering applications, ${ }^{35-36}$ and this approach has been also applied in $3 \mathrm{D}$ printing technology. ${ }^{37}$ Increasing studies have indicated that superparamagnetic nanoparticles embedded in scaffolds can still be magnetized under magnetic fields, which allow the scaffolds show the superparamagnetic performance ${ }^{9,38}$ that has been demonstrated promotion of osteogenesis in vitro and in vivo., ${ }^{5,6,8,10,31}$ However, whether the superparamagnetic property is associated with polymer chemistry and physical morphology is still unkonwn. In addition, the relation between the effect of osteogenic promotion and the superparamagnetic property of various scaffolds has been little touched as well.

In this work, there were 9 kinds of scaffold in the present or absent magnetic field were set for comparably investigating the difference of the scaffolds in the osteogenic enhancement. We aimed to compare the scaffolds in two aspects. One is that scaffolds with the same morphology but different polymeric components (PLA, $\mathrm{PU}$, and gelatin) that are three widely used in the tissue engineering and regenerative medicine, while their mechanical properties are different, to compare the role of chemical composition of scaffolds. The other aspect is that scaffolds with the same polymeric component but different morphology (fiber, porous, or film), to observe the role of physical features of scaffolds.

The results showed that the magnetization for scaffolds was associated with both the chemical composition and physical microstructure. The difference might be partly resulted from the mechanical properties of the polymers. In our opinions, when MNPs are embedded in the polymeric substrate, for the high ratio of atoms on the surface, ${ }^{39}$ their superparamagnetic property is dependent on the chemical environment where they are locating, that means the polymer molecules surrounding the MNPs would affect the superparamagnetic of MNPs, which results in different magnetization of scaffolds fabricated with different polymers. At the same time, the chemical composition might have influence on the

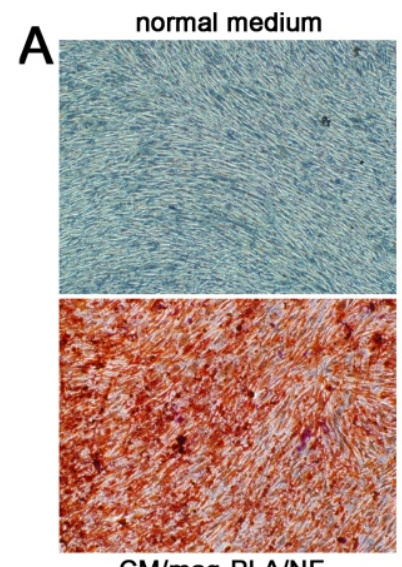

$\mathrm{CM} /$ mag-PLA/NF inductive medium

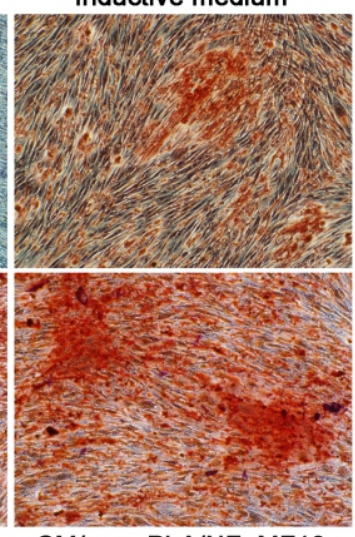

$\mathrm{CM} /$ mag-PLA/NF+MF10

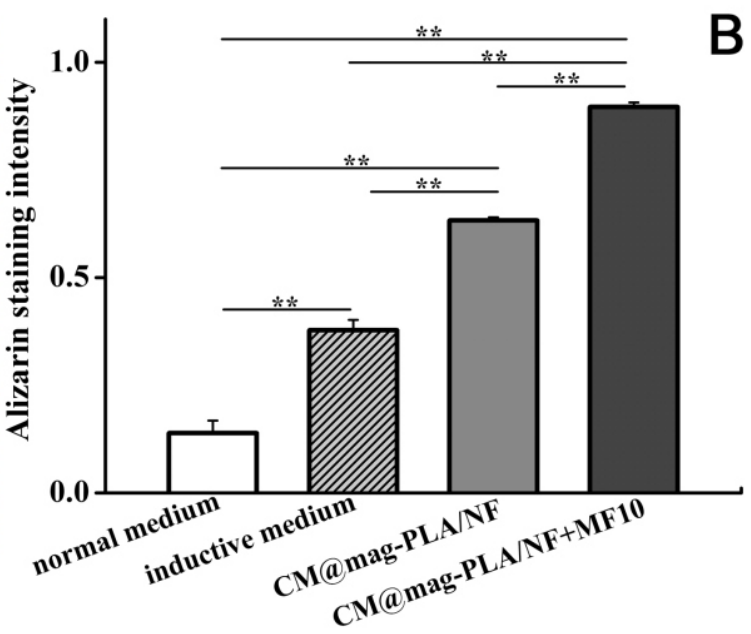

Fig. 8 The differentiation of MSCs induced by the conditioned media. (A) Alizarin red staining of MSCs incubated with different media for 21 days. (B) Quantitative results of (A). 
dispersion of MNPs embedded in the scaffolds as well as determined the mechanical properties of scaffolds. PLA is one plastic material with relative higher mechanical strength than PU, while PU is highly elastic, and Gel is one soft hydrogel. It was shown that MNPs incorporated in the plastic PLA generated much stronger superparamagnetic response, while those in the hydrogel of gelatin generated weak response to the magnetic filed. At the same time, cells grown on mag-PLA exhibited higher degree of differentiation than those on mag-PU and mag-Gel. Therefore we would suggest that among the three kinds polymers, mag-PLA was more potent in the enhancement of osteogenesis. These results provided important cues in the design of superparamagnetic scaffolds for guiding tissue regeneration in different demands, though the physical mechanisms of how matrix materials affect the magnetization behavior still require further investigation. Nevertheless, there are limitations in this study. There are quite many physical and chemical factors for the scaffolds characterization, and also many biological markers that reflect the osteogenesis process, which would be a huge and complicated system for the study. Hence we could only selected one magnetic field strength and three kinds of polymeric material in the cellular experiments. In addition, due to the technical limitation, the mechanical force that applied to the cells by the different scaffolds was not able to measure directly, which was characterized by the magnetization in this study.

Considering scaffolds with $10 \%$ MNPs (w \% of polymer) was demonstrated one optimal formulation in our previous work, ${ }^{9}$ and also used in other studies, ${ }^{40-41} 10 \%$ MNPs was selected in this study for all cell assays to avoid the study system too complicated. However, it should be noticed that changing the concentration of MNPs in the scaffold could modulate the magnetization property of scaffolds and their enhancement function of osteogenesis. For examples, porous PCL with $10 \%$ MNPs exhibited higher level of saturated magnetization and stronger enhancement effect on osteogenesis than that with $5 \%$ MNPs; ${ }^{6}$ nanofibrous PCL with $20 \%$ MNPs showed higher magnetization and promoted more significant odontogenesis of human dental pulp cells, as well as the angiogenesis of HUVECs than that with $10 \% \mathrm{MNPs},{ }^{42}$ nanofibrous PLA with $5 \%$ MNPs could further increase the ALP activity of MC3T3-E1 cells, along with the calcium content than that with $2.5 \%$ MNPs; ${ }^{7}$ porous hydroxyapatite (HA) with $2 \%$ MNPs displayed the best promotion effect in osteogenesis of MC3T3-E1 cells than that with $1.5 \%, 1 \%$ or $0.5 \%$ MNPs groups. ${ }^{43}$

It has been known that macrophages and fibroblasts are involved in all phases of the natural process of wound healing, which are responsible for the balance of environment and remodeling. ${ }^{44-46}$ Our previous research demonstrated that mag-PLA/NF+MF enhanced the secretion of growth factors for both macrophages and fibroblasts, including VEGF, PDGF and $\mathrm{bFGF}^{9,15}$ In this work, we showed that the enhanced osteogenic differentiation of bMSCs might due to the increased secretion of growth factors. The conditioned medium could increase the production of ECM and mineralization remarkably, the inductive effect was even higher than that of the pure inductive medium. The enhancement of interactions among macrophages, fibroblasts and bMSCs mimics the natural microenvironment, and provide side evidence that the superparamagnetic scaffolds could enhance the osteogenesis process, not only by interacting with pre-osteoblasts and bMSCs, but also by interacting with macrophages and fibroblasts, and enhancing these different cells cross talk, which will be beneficial for the bone tissue regeneration.

\section{Conclusions}

In summary, for the current composite samples, the magnetization behavior was closely associated with the chemical composition and physical microstructure of scaffolds, which modulated the guidance of osteogenic differentiation. The higher the saturated magnetization was, the stronger the promotion to the osteogenesis. Under the static magnetic field, the mag-PLA/NF showed the highest saturated magnetization among the 9 scaffolds and the strongest promotion to the osteogenic differentiation of pre-osteoblasts and bMSCs, and additionally enhanced the cross talk of bMSCs with macrophages and fibroblasts in the osteogenesis. Nevertheless, parameters investigated and compared in this study are far of adequate, therefore more chemical compositions, structural features and magnetic field intensity should be included in the future work. In addition, it would be better to measure the mechanical stimuli that were generated by the scaffolds instead of characterizing them using the saturated magnetization.

\section{Acknowledgment}

This work was supported by National Natural Science Foundation of China (NSFC 81471793) and CAMS Innovation Fund for Medical Sciences (CIFMS 2016-I2M -3-004).

\section{References}

1. P. V. Giannoudis, H. Dinopoulos and E. Tsiridis, Injury, 2005, 36, S20-S27.

2. C. Laurencin, Y. Khan and S. F. El-Amin, Expert Rev. Mol. Med., 2006, 3, 49-57.

3. C. Szpalski, M. Wetterau, J. Barr and S. M. Warren, Tissue Eng. Part B-Rev., 2012, 18, 246-257.

4. A. R. Amini, D. J. Adams, C. T. Laurencin and S. P. Nukavarapu, Tissue Eng. Part A, 2012, 18, 1376-1388.

5. J. Meng, Y. Zhang, X. Qi, H. Kong, C. Wang, Z. Xu, S. Xie, N. $\mathrm{Gu}$ and H. Xu, Nanoscale, 2010, 2, 2565-2569.

6. H. M. Yun, S. J. Ahn, K. R. Park, M. J. Kim, J. J. Kim, G. Z. Jin, H. W. Kim and E. C. Kim, Biomaterials, 2016, 85,88-98.

7. Q. Cai, Y. Shi, D. Shan, W. Jia, S. Duan, X. Deng and X. Yang, Mater. Sci. Eng. C-Mater. Biol. Appl., 2015, 55, 166-173.

8. J. Meng, B. Xiao, Y. Zhang, J. Liu, H. Xue, J. Lei, H. Kong, Y. Huang, Z. Jin, N. Gu and H. Xu, Sci. Rep., 2013, 3, 2655.

9. S. Hao, Y. Zhang, J. Meng, J. Liu, T. Wen, N. Gu and H. Xu, ACS Appl. Mater. Interfaces, 2018, 10, 22913-22923.

10. S. Panseri, A. Russo, M. Sartori, G. Giavaresi, M. Sandri, M. Fini, M. C. Maltarello, T. Shelyakova, A. Ortolani, A. Visani, V. Dediu, A. Tampieri and M. Marcacci, Bone, 2013, 56, 432-439.

11. H. Markides, J. S. McLaren, N. D. Telling, N. Alom, E. A. AlMutheffer, R. O. C. Oreffo, A. Zannettino, B. E. Scammell, L. J. White and A. J. El-Haj, NPJ Regen. Med., 2018, 3, 9.

12. Y. Sapir-Lekhovitser, M. Y. Rotenberg, J. Jopp, G. Friedman, B. Polyak and S. Cohen, Nanoscale, 2016, 8, 3386-3399.

13. R. Fuhrer, S. Hofmann, N. Hild, J. R. Vetsch, I. K. Herrmann, R. N. Grass and W. J. Stark, PLoS One, 2013, 8, e81362.

14. R. K. Singh, K. D. Patel, J. H. Lee, E. J. Lee, J. H. Kim, T. H. Kim and H. W. Kim, PLoS One, 2014, 9, e91584.

15. S. Hao, J. Meng, Y. Zhang, J. Liu, X. Nie, F. Wu, Y. Yang, C. Wang, N. Gu and H. Xu, Biomaterials, 2017, 140, 16.

16. Y. Wei, X. Zhang, Y. Song, B. Han, X. Hu, X. Wang, Y. Lin and X. Deng, Biomed. Mater., 2011, 6(5), 055008. 
17. J. Zhang, S. Zhao, M. Zhu, Y. Zhu, Z. Liu and C. Zhang, J. Mater. Chem., 2014, 2(43), 7583-7595.

18. J. Huang, Y. Liang, Z. Jia, J. Chen, L. Duan, W. Liu, F. Zhu, Q. Liang, W. Zhu, W. You, J. Xiong and D. Wang, ACS Omega, 2018, 3, 6182-6189.

19. E. D. Silva, P. S. Babo, R. Costa-Almeida, R. M. A. Domingues, B. B. Mendes, E. Paz, P. Freitas, M. T. Rodrigues, P. L. Granja and M. E. Gomes, Nanomedicine, 2017, 14, 2375-2385.

20. C. Ribeiro, V. Correia, P. Martins, F. M. Gama and S. LancerosMendez, Colloids Surf. B, 2016, 140, 430-436.

21. R. De Santis, A. Gloria, T. Russo, U. D'Amora, S. Zeppetelli, C. Dionigi, A. Sytcheva, T. Herrmannsdörfer, V. Dediu and L. Ambrosio, J. Appl. Polym. Sci., 2011, 122, 3599-3605.

22. J. W. Lu, F. Yang, Q. F. Ke, X. T. Xie and Y, P. Guo, Nanomedicine, 2018, 14, 811-822.

23. D. Shan, Y. Shi, S. Duan, Y. Wei, Q. Cai and X. Yang, Mater. Sci. Eng. C, 2013, 33, 3498-3505.

24. F. Yang, J. Lu, Q. Ke, X. Peng, Y. Guo and X. Xie, Sci. Rep., 2018, 8, 7345.

25. M. Bianchi, M. Boi, M. Sartori, G. Giavaresi, N. Lopomo, M. Fini, A. Dediu, A. Tampieri, M. Marcacci and A. Russo, J. Mater. Sci. Mater. Med., 2015, 26(1), 1-9.

26. S. Hu, Y. Zhou, Y. Zhao, Y. Xu, F. Zhang, N. Gu, J. Ma, M. A. Reynolds, Y. Xia and H. H. K. Xu, J. Tissue Eng. Regen. Med., 2018, 12(4), e2085-e2098.

27. A. Russo, M. Bianchi, M. Sartori, M. Boi, G. Giavaresi, D. M. Salter, M. Jelic, M. C. Maltarello, A. Ortolani, S. Sprio, M. Fini, A. Tampieri and M. Marcacci, J. Biomed. Mater. Res. B Appl. Biomater., 2018, 106, 546-554.

28. A. Tampieri, E. Landi, F. Valentini, M. Sandri, T. D'Alessandro, V. Dediu and M. Marcacci, Nanotechnology, 2011, 22, 015104.

29. Y. Wu, W. Jiang, X. Wen, B. He, X. Zeng, G. Wang and Z. Gu, Biomed. Mater., 2010, 5, 015001.
30. Z. P. Chen, Y. Zhang, S. Zhang, J. Xia, J. Liu, K. Xu and N. Gu, Colloids Surf. A, 2008, 316(1), 210-216.

31. K. Dashnyam, R. A. Perez and R. K. Singh, RSC Advances, 2014, 4, 40841-40851.

32. M. S. Rahman, N. Akhtar, H. M. Jamil, R. S. Banik and S. M. Asaduzzaman. Bone Res., 2015, 14, 15005.

33. V. I. Sikavitsas, J. S. Temenoff and A. G. Mikos, Biomaterials, 2001, 22, 2581-2593.

34. A. W. Orr, B. P. Helmke and B. R. Blackman, Dev. Cell, 2006, 10, 11-20.

35. A. Ortolani, M. Bianchi, M. Mosca, S. Caravelli, M. Fuiano, M. Marcacci and A. Russo, Joints, 2017, 4, 228-235.

36. H. Xu and N. Gu, Front. Mater. Sci., 2014, 8(1), 20-31.

37. I. A. Paun, R. C. Popescu, B. S. Calin, C. C. Mustaciosu, M. Dinescu and C. R. Luculescu, Int. J. Mol. Sci., 2018, 19, 495.

38. S. L. Arias, A. Shetty, J. Devorkin and J. P. Allain, Acta Biomater., 2018, 77, 172-181.

39. B. Issa, I. M. Obaidat, B. A. Albiss and Y. Haik, Int. J. Mol. Sci., 2013, 14, 21266-21305.

40. Z. Liu, L. Huang, L. Liu, B. Luo, M. Liang, Z. Sun, S. Zhu, X. Quan, Y. Yang, T. Ma, J. Huang and Z. Luo, Int. J. Nanomed., 2014, 10, 43-61.

41. R. De Santis, U. D'Amora, T. Russo, A. Ronca, A. Gloria and L. Ambrosio, J. Mater. Sci. Mater. Med., 2015, 26, 250.

42. H. M. Yun, S. K. Kang, R. K. Singh, J. H. Lee, H. H. Lee, K. R. Park, J. K. Yi, D. W. Lee, H. W. Kim and E. C. Kim, Dent. Mater., 2016, 32(11), 1301-1311.

43. X. B. Zeng, H. Hu, L. Q. Xie, F. Lan, W. Jiang, Y. Wu and Z. W. Gu, Int. J. Nanomed., 2012, 7, 3365-3378.

44. T. A. Wynn and K. M. Vannella, Immunity, 2016, 44, 450-462.

45. M. L. Novak and T. J. Koh, J. Leukocyte Biol., 2013, 93, 875.

46. C. Wiegand and R. White, Wound Repair Regen., 2013, 21, $793-$ 799. 